\title{
Materials FA Investigation of a Semiconductor Laser Diode package with SEM FIB EDS and correlation with the Feedback Oxidation Loop of FA literature
}

\author{
Clara Podpora*, Mark McElhinney**, and Robert Walker *** \\ Lasertel, Inc., Tucson, Arizona. *MACFA Consultant, **President, ***VP
}

Introduction. It had been determined by product design engineers, after reliability testing, that three laser diode devices had exhibited a "low power" state. A Materials Failure Analysis investigation was performed to determine if the condition had originated on site, in manufacturing, or at the customer's facility. Progressive macroscopic to microscopic methods were utilized and included Optical Microscopy, SEM, EDS, and FIB, respectively.

SEM investigation of the front facet coating of the semiconductor laser bar revealed an "oval" defect (Fig.1). This defect is shaped in the manner of the emitted light, and is aligned with the active epitaxial region. FIB cross sectioning was chosen in order to gradually expose the interface of the facet coating and semiconductor laser bar at the defect region. The resulting FIB trench showed that not one, but two areas of material alteration existed. First, the oval defect, which was discovered originally with the SEM, was shown by FIB to definitively be within the facet coating. Small voids were found beneath the surface of the oval defect. A second feature, named the "comet tail" defect (Fig. 2), was discovered extending backward into the semiconductor laser bar.

The physical proximity of these material defects posed the question: Which occurred first? Three types of material defect mechanisms are probable: 1) that described in this report; 2) surface contamination; or 3) electromigration of solder, and all could result in a similar looking oval defect. By observing the interior of the semiconductor via FIB, defect mechanisms $2 \& 3$ were excluded. Utilization of SEM /FIB /EDS along with the Oxidation Feedback Loop [1] (Fig.3) helped to determine the material point source of the mechanism that caused the material to change from the intended product design.

"Oval" Facet Coating Defect. There are two possible points of origin for the degradation process that produced the oval facet coating defect: 1) a condition of non-ideal composition within the deposited coating layer, or, 2) at the facet coating /laser bar interface. Both of these points of origin would have the end result of altering the physical and chemical protective properties of the facet coating.

"Comet Tail" Defect. The "comet tail" feature is altered semiconductor laser bar material. It exists within the active epitaxial region. Oxidized semiconductor material will show up lighter in atomic number contrast in the SEM [1]. Laser bar facet oxidation is the breakdown of the original epitaxial compounds of the semiconductor. EDS elemental analysis performed on the defect, both unsectioned and FIB-sectioned, show a depletion of arsenic (Fig 5a,c). Arsenic oxidizes most easily of all the elements present in a semiconductor crystal design, and is also the most mobile [1] [2].

Chronological Relationship of the defects. The heat from the laser light caused a localized physical-chemical change within a specific area of non-ideal facet coating. The simultaneous composition and volume change caused both the oval shape and the small voids present below the surface. As the coating was altered, oxygen, heat, and light to the semiconductor laser bar increased, 
causing both oxidation and non-radiative recombination to propagate. This active process caused the chemical changes that advanced into the laser bar crystal, and is evidenced by both the comet tail defect and the depletion of arsenic concentration. Light absorption at the facet, electron-hole pair generation, non-radiative recombination, heating, and facet oxidation all play a role, as the dynamic material mechanisms of the Oxidation Feedback Loop and COD [1]. Shown here are the very beginning stages of this loop.

In many of these cases only the final step, i.e.; catastrophic melting of laser the bar, is observed. When this has occurred, the details of the failure mechanism have often been erased due to the intermixing of several component materials. Because as few as three alternative defect mechanisms will cause a similar catastrophic meltdown, locating the exact point source mechanism of the material degradation is essential. In this case, with the FIB, the point source was found to be at the facet coating /laser bar interface. Comprehensive materials failure analysis provided the product engineers with the very specific data required for their design and manufacturing solutions.

\section{References:}

[1] M. Fukudo, Reliability and Degradation of Semiconductor Lasers and LEDs, Artech House, $1^{\text {st }}$ ed., Boston-London, 1991.

[2] O. Ueda, Reliability and Degradation of III-V Optical Devices, Artech House, $1^{\text {st }}$ ed., BostonLondon, 1996.

[3] N.N. Greenwood and A. Earnshaw, Chemistry of the Elements, Pergamon Press, $4^{\text {th }}$ ed., OxfordNew York, 1989.

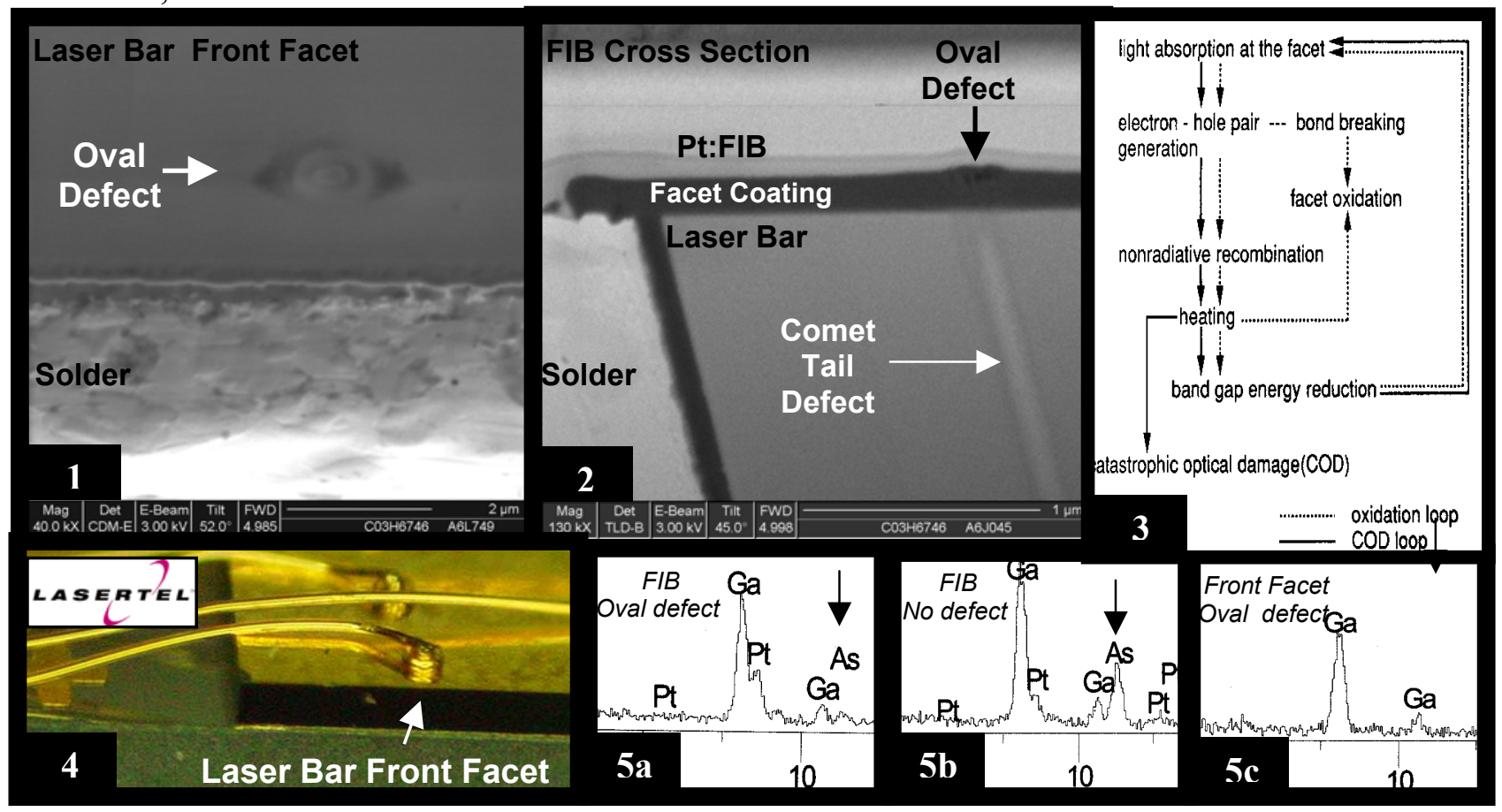

Fig. 1 SEM: Oval Defect on laser bar front facet. Fig. 2 FIB: Trench shows Comet Tail Defect.

Fig. 3 Oxidation Feedback Loop: M. Fukudo [1]. Fig. 4 Optical Microscope image of laser device. Fig. 5 As depletion: 5a. EDS: oval defect, 5b. EDS: area w/out defect, 5c. EDS: front facet w/defect. 